\title{
Grasping Neurons of Monkey Parietal and Premotor Cortices Encode Action Goals at Distinct Levels of Abstraction during Complex Action Sequences
}

\author{
Luca Bonini, ${ }^{1}$ Francesca Ugolotti Serventi, ${ }^{1}$ Luciano Simone, ${ }^{1}$ Stefano Rozzi, ${ }^{1}$ Pier Francesco Ferrari, ${ }^{1,2}$ \\ and Leonardo Fogassi ${ }^{1,3}$ \\ ${ }^{1}$ Dipartimento di Neuroscienze, Università di Parma and Istituto Italiano di Tecnologia, Brain Center for Motor and Social Cognition, 43125 Parma, Italy, \\ ${ }^{2}$ Dipartimento di Biologia Evolutiva e Funzionale, Università di Parma, 43124 Parma, Italy, and ${ }^{3}$ Dipartimento di Psicologia, Università di Parma, 43121 \\ Parma, Italy
}

\begin{abstract}
Natural actions are formed by distinct motor acts, each of which is endowed with its own motor purpose (i.e., grasping), chained together to attain the final action goal. Previous studies have shown that grasping neurons of parietal area PFG and premotor area F5 can code the goal of simple actions in which grasping is embedded. While during simple actions the target is usually visible, directly cueing the final goal, during complex action sequences is often concealed and has to be kept in mind to shape action unfolding. The aim of this study was to assess the relative contribution of sensory-cued or memory-driven information about the final goal to PFG and F5 grasping neurons activity. To this purpose, we trained two monkeys to perform complex action sequences, each including two successive grasping acts, aimed at specific final goals (eating or placing). We recorded 122 PFG and 89 F5 neurons. Forty-seven PFG and 26 F5 neurons displayed action goal selectivity only during the late phase of the action, when sensory information cueing the action goal became available. Reward contingency did not affect neuronal selectivity. Notably, 17 PFG neurons reflected the final goal from the early phase of action unfolding, when only memory-driven information was available. Crucially, when monkeys were prevented from obtaining such information before action onset, neurons lost their early selectivity. Our findings suggest that external sensory cues and individual's motor intention integrate at different level of abstraction within a large anatomo-functional network, encompassing parietal and premotor cortices.
\end{abstract}

\section{Introduction}

Human and nonhuman primates are capable of performing intentional actions to different degrees of complexity (Koechlin and Jubault, 2006; Botvinick, 2007; Fuster, 2008). Simple natural actions are formed by a brief sequence of motor acts (such as reaching, grasping, and bringing to the mouth) chained to each other to attain the final goal of the whole action (e.g., eating, drinking). Neurophysiological studies have shown that the discharge of neurons in the ventral premotor (Rizzolatti et al., 1988; Kakei et al., 2003; Umiltà et al., 2008) and inferior parietal (Gardner et al., 2007; Rozzi et al., 2008) cortices encodes the goal of single motor acts, the basic building blocks for organizing actions. In addition to coding single acts, grasping neurons in the inferior parietal area PFG (as defined by Gregoriou et al., 2006) and, to a less extent, in the ventral premotor area F5 (as defined by

Received Oct. 4, 2010; revised Feb. 17, 2011; accepted Feb. 25, 2011.

Author contributions: L.B., S.R.,P.F., and L.F. designed research; L.B., F.U.S., and L.S. performed research; L.B. and F.U.S. analyzed data; L.B., S.R., P.F., and L.F. wrote the paper.

The work was supported by Italian Space Agency (PR-DCMC-G0-1B1125-003), Minestero dell' Istruzione, dell' Universita e della Ricerca (2004057380 and 2006052343), the European Commission grant Cogsystems (FP7250013) and Italian Institute of Technology. We thank H. Scherberger and V. Gallese for their valuable comments on an early version of the manuscript.

Correspondence should be addressed to Dr. Luca Bonini, Università di Parma and Istituto Italiano di Tecnologia, Brain Center for Motor and Social Cognition, via Volturno 39, 43125 Parma, Italy. E-mail: luca.bonini@unipr.it.

DOI:10.1523/JNEUROSCI.5186-10.2011

Copyright $\odot 2011$ the authors $\quad 0270-6474 / 11 / 315876-12 \$ 15.00 / 0$
Matelli et al., 1985), can discharge differently according to the final goal (i.e., eating or placing) of the action in which the coded act is embedded (Fogassi et al., 2005; Bonini et al., 2010). This parietofrontal circuit appears to be crucial for organizing distinct motor acts into actions (Fuster, 2006; Rizzolatti et al., 2006; Jubault et al., 2007).

The organization of simple actions often relies on sensory information associated with the target (Toth and Assad, 2002) in a given environmental context (Salinas, 2004; Baumann et al., 2009) for selecting the final goal. For example, the vision of a steaming cup of tea is a sensory cue that suggests the observer drink it, whereas viewing the same cup, empty after the teatime, prompts one to clean it up. In contrast, longer and more complex action sequences performed in our daily life often do not rely only on such a sensory-cued selection of the action goal because the target cueing the final goal may not be available before action onset. Thus, the agent has to form an internal representation of the goal and keep it in mind to shape action unfolding. For example, to eat a candy, one needs to open the candy box, grasp the candy, and eat it. In this case, the first part of the action sequence (opening the box) is memory-driven because the agent has to know that the candy is in the box, although it is not visible, and use this knowledge for action planning.

The present study aims at investigating whether, during complex actions, the discharge of parietal and premotor neurons can reflect the final action goal only when it is sensory-cued (target 
A

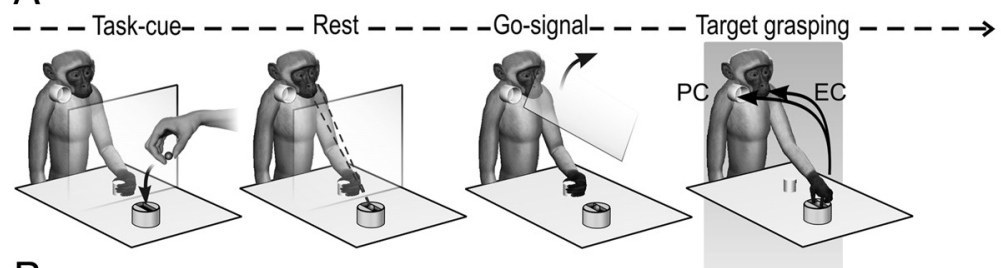

B

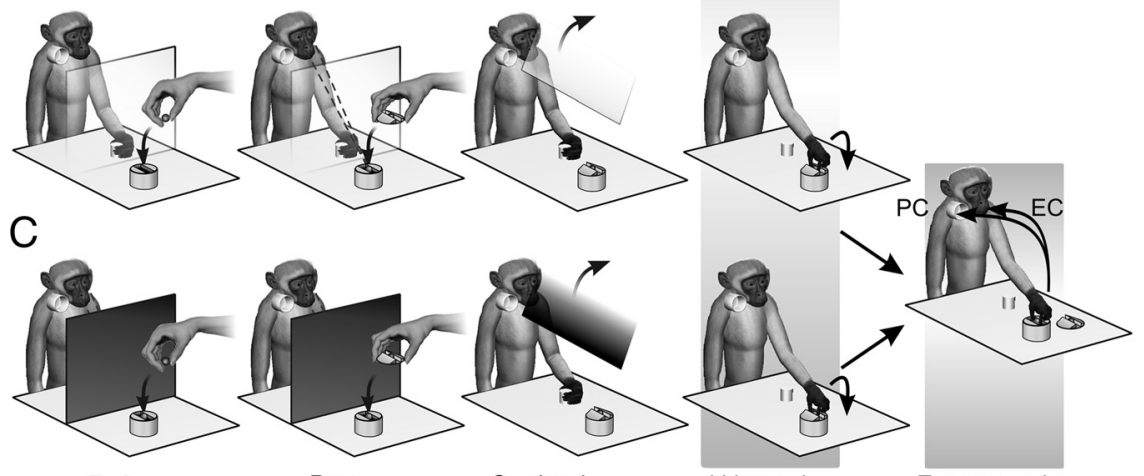

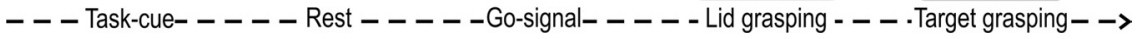

Figure 1. Action sequence tasks. $\boldsymbol{A}$, Simple action. $\boldsymbol{B}$, Complex action with the transparent screen, which allowed the monkey to establish from the beginning which of the two conditions (eating or placing) had to be performed. C, Complex action with the opaque screen, which prevented the monkey from seeing the final target of the ongoing action and thus from establishing which of the two conditions had to be performed. EC, Eating condition; PC, pacing condition. See Materials and Methods for detailed description of the two tasks.

visible), or also when it is memory-driven (target hidden). To this purpose, we recorded the discharge of PFG and F5 grasping neurons while monkeys performed complex natural action sequences aimed at distinct final goals (either eating or placing). Each sequence included two grasping acts differing from each other in terms of availability of sensory-cued or memory-driven information about the final goal.

\section{Materials and Methods}

The experiments were performed on two female macaque monkeys (Macaca nemestrina).

Before recordings, monkeys were habituated to sit in a primate chair and to interact with the experimenters. Then they were trained to perform the motor tasks described below using the hand contralateral to the hemisphere to be recorded. When the training was completed, a head fixation system and a titanium recording chamber were implanted under general anesthesia (ketamine hydrocloride, $5 \mathrm{mg} / \mathrm{kg}$, i.m. and medetomidine hydrocloride, $0.1 \mathrm{mg} / \mathrm{kg}$, i.m.), followed by postsurgical pain medications. Surgical procedures were the same as previously described (Rozzi et al., 2006; Bonini et al., 2010). All the experimental protocols were approved by the Veterinarian Animal Care and Use Committee of the University of Parma and complied with the European law on the humane care and use of laboratory animals.

Behavioral tasks and apparatus. Both monkeys were trained to perform two distinct action sequence tasks of different degree of complexity, which we will refer to as the complex action and the simple action. Each of the two tasks included the same two experimental conditions, namely eating and placing.

Simple action. This task (Fig. $1 \mathrm{~A}$ ) has been previously applied to study inferior parietal and ventral premotor grasping neurons activity, and the details about the experimental set-up and paradigm have been described previously (Fogassi et al., 2005; Bonini et al., 2010). In brief, the monkey held its hand in a fixed position while the experimenter positioned the target item [a piece of food (a $1 \times 1 \mathrm{~cm}$ piece of apple, carrot, or potato) or an object (of the same size and shape of the food)] in a container (Fig. $1 \mathrm{~A}$, Task-cue). The target item was located within a rectangular groove ( $40 \times 12 \mathrm{~mm}$; depth, $10 \mathrm{~mm}$ ) carved into the container (height, $5 \mathrm{~cm}$ ) to force the monkey to always adopt the same type of grip (precision grip). During these operations, preliminary to the forthcoming trial, there was a transparent screen between the monkey and the target. The first part of the task was identical in the two experimental conditions: after screen removal (Fig. $1 A$, Gosignal), the monkey reached and grasped the target item using a precision grip (Fig. $1 A$, Target grasping). In the eating condition, the monkey had to bring the food to the mouth and eat it; in the placing condition, it had to place the object (or food) into a container located near the mouth to obtain a reward that was delivered after a variable and unpredictable time lag ( $1-4 \mathrm{~s})$. When placing of the food was required, a more palatable food reward was presented for a brief period of time (1-2 s) (Fig. $1 \mathrm{~A}$, Rest) before trial onset, to instruct the monkey to place the food morsel instead of eating it. After a variable time lag $(2-5 \mathrm{~s})$, the screen was lifted and the monkey was allowed to perform the action. After correct accomplishment of the task, the monkey either ate the grasped food (eating condition) or, in placing condition, it was rewarded with either a food morsel identical to that obtained in the eating condition (for placing an object) or a more palatable food reward (for placing food).

Complex action. The experimental set-up and basic procedure were the same as those used for the simple action, but the container in which the target was located was covered with a lid. Thus, to accomplish the task, the monkey had to perform two successive grasping acts. The lid had a small handle located in the center of a groove $(40 \times 12 \times 10 \mathrm{~mm})$; this feature forced the monkey to grasp the handle with a precision grip identical to that used for the successful grasping of the target (see Simple action, above). At the beginning of the task, the monkey held its hand on a fixed position behind a transparent plastic screen while the experimenter positioned the target (a piece of food or an object of the same size and shape of the food) in the container and covered it with the lid (Fig. $1 B$, Task-cue). The use of the transparent screen allowed the monkey to see what would be the final target (food or object) of the second grasping act. As described for the simple action, when the target of the placing condition was the same food as that used in the eating condition, the monkey was presented with a more palatable reward for a brief period of time (1-2 s) (Fig. 1 B, Rest) to instruct it to place the food morsel instead of eating it. Then, after a variable time lag $(2-5 \mathrm{~s})$, the screen was removed (Fig. $1 B$, Go-signal). The monkey reached for and grasped (with a precision grip) the handle of the lid and removed it (Fig. $1 B$, Target grasping). Then, it grasped (with a precision grip) the food or the object located inside (Fig. $1 \mathrm{~B}$, far right) and brought the food to the mouth and ate it (eating condition) or placed the object or food morsel into a container located near the mouth (placing condition). Note that the two conditions are motorically identical until target grasping, and only after this act do they diverge. It is also important to note here that from target grasping to the end of the action sequence, the complex action is identical to the corresponding phase of the simple action in each condition (eating or placing conditions). The reward contingency associated with correct task accomplishment was the same as in the simple action.

The complex action was used for studying all the neurons included in the present dataset and the two experimental conditions (eating and placing conditions) were run in a random fashion. Whenever both complex and simple actions were applied, they were run together in a random fashion.

In a further experiment, the monkeys performed a modified version of the complex action in which the Go-signal was provided by means of an opaque, rather than transparent, screen (Fig. 1C). This prevented the monkey from establishing the final goal of the action to be performed from the beginning. Some neurons were recorded in this version of the 
complex action by running the two experimental conditions in blocks. Note that, compared with the transparent-screen version of the complex action, in this version no cue was available before trial onset (Task-cue period) to indicate which action had to be performed.

In both the simple and complex actions, if the monkey detached its hand from the starting cylinder before the go signal or failed to correctly grasp the lid or the target, the trial was discarded and not included in the dataset. In particular, if the monkey ate the food when it was used as the target in the placing condition, the trial was discarded and reward was not delivered. Deleted trials were repeated to collect at least 10 trials for each experimental condition.

Recording techniques. Neuronal recordings were performed by means of single glasscoated microelectrodes (impedance, 0.5-1 $\mathrm{M} \Omega$ ) inserted through the intact dura. The microelectrode was mounted on an electrode holder and connected to a computer-controlled microdrive. Dedicated software (EPS; Alpha Omega) controlled the engine for electrode movement. The electrode holder was attached to a stereotaxic arm, mounted on the monkey-head holder. Neuronal activity was amplified and monitored on an oscilloscope. Single-neuron action potentials were isolated with a dual voltage-time window discriminator (Bak Electronics) and fed to a personal computer as digital signals to be recorded, stored, and subsequently analyzed.

Clinical testing of the recorded neurons. Once a neuron was isolated, its motor, visual, and somatosensory properties were tested (for testing procedures, see Fogassi et al., 2005; Rozzi et al., 2008). Neurons showing strong somatosensory responses were excluded from further testing with the motor tasks. These preliminary tests also allowed identification of possible responses related to mouth or arm motor acts. The relationship between neuronal responses and hand or mouth grasping was established by testing each effector independently. Hand grasping was tested with the arm restrained, so that only hand movements were allowed. Mouth grasping was tested by restraining the monkey's arm and placing pieces of food directly into the monkey's mouth. Neurons exclusively active during arm-related motor acts (such as reaching or bringing to the mouth) or during mouth grasping were not included in this study. Neurons responsive to grasping with both hand and mouth were analyzed separately. Only neurons showing a motor response during hand grasping performed with a precision grip were considered for testing with the complex action.

Those neurons that fulfilled the requirements for study with the complex action were also preliminary tested by means of the simple action using different types of food, to exclude the possibility that differences in terms of reward could explain the possible differential discharge between the experimental conditions. Furthermore, during preliminary test with the simple action, all the neurons included in the final dataset did not show any response to visual presentation of objects or sustained activity during preparatory period after cue presentation and while waiting for the go signal.

Recording of behavioral events and definition of grasping epochs. Contact-detecting electric circuits were used to provide digital signals related to the main behavioral events: (1) detachment of the hand from the starting point, (2) contact of the hand with the handle of the lid (in the complex action only), (3) contact of the hand with the food or object target of the action, and (4) contact of the hand with the border of the container in which the object/food had to be placed (in the placing condition of both tasks). These signals were subsequently used to align neuronal activity in different trials and to construct the response histograms and the data files for statistical analysis. All the data were acquired and stored by means of LabView software, allowing us to record neuronal activity aligned with the corresponding events of the behavioral paradigm.

Based on digital signals related to the behavioral events described above, we defined three epochs of interest in which neuronal activity was compared: (1) baseline, starting $2 \mathrm{~s}$ before the hand detached from the starting position and lasting $600 \mathrm{~ms}$ (this epoch applies to both the simple and the complex actions); (2) epoch 1, starting $250 \mathrm{~ms}$ before the hand contacted the handle of the lid and ending $160 \mathrm{~ms}$ after; this epoch was also used for the analysis of target-grasping activity in the simple action; and (3) epoch 2, starting $120 \mathrm{~ms}$ before the hand contacted the target object to $160 \mathrm{~ms}$ after (second grasping act in the complex action). Time intervals of epoch 1 in both the simple and complex actions have been chosen with a largely accepted, broad definition of hand-grasping motor act, which includes both the hand shaping and actual grasping phases (Jeannerod, 1988; Mason et al., 2004; Chen et al., 2009). Note that epochs 1 and 2 have been used for analyzing grasping activity with respect to baseline and to compare grasping activity between the two conditions (eating and placing), not for comparing the epochs or the tasks. To compare grasping activity and action goal selectivity across epochs and tasks (complex and simple action), we considered grasping epochs of the same duration (that of epoch 2) for grasping both the lid and the target.

Data analyses. The activity of each neuron (10 trials) has been considered in terms of mean firing rate (spikes/s) in different epochs according to the task (simple or complex action). Single-neuron response in the simple action was statistically assessed by mean of a $2 \times 2$ ANOVA for repeated measures (factors, condition and epoch), followed by Newman-Keuls post hoc tests. The responses of the same neurons recorded in the complex action were statistically assessed by mean of a $2 \times 3$ repeatedmeasures ANOVA (factors, condition and epoch), followed by NewmanKeuls post hoc tests. All analyses were performed using a significance criterion of $p<0.05$. Only neurons significantly activated during at least one of the grasping acts in one of the two tasks have been included in this study. All neurons showing a significantly different discharge rate between the two experimental conditions (eating and placing) during one of the grasping epochs were considered as action goal-related (AGR), as previously described (Fogassi et al., 2005; Bonini et al., 2010).

To quantitatively assess the degree of preference expressed by single neurons for eating or placing conditions, a preference index (PI) was calculated as follows: $\mathrm{PI}=\left(R_{\mathrm{e}}-R_{\mathrm{p}}\right) /\left(R_{\mathrm{e}}+R_{\mathrm{p}}\right)$, where $R_{\mathrm{e}}$ and $R_{\mathrm{p}}$ are the average responses of the neuron in the eating and placing conditions, respectively. The PI values range from -1 (complete selectivity for placing condition) to 1 (complete selectivity for eating), and a value of zero corresponds to identical discharges in the eating and placing conditions.

Population analyses have been performed, taking into account single neurons responses expressed in terms of net-normalized mean activity (Bonini et al., 2010). For each neuron, the mean activity was calculated in $20 \mathrm{~ms}$ bins in all the recorded trials of the experimental conditions to be compared. Then, for each condition, an off-set procedure was applied, subtracting the mean baseline activity from the value of each bin (net activity). The highest net activity value among the compared conditions was used to divide the value of each single bin (net-normalized mean activity). In this way, each neuron, regardless of its original firing rate, was characterized by a mean baseline activity equal to 0 and a peak activity value of 1 .

Histological reconstruction and identification of the recorded regions. Approximately 1 week before sacrificing the animals, we performed elec- 
trolytic lesions ( $10 \mu \mathrm{A}$ cathodic pulses per $10 \mathrm{~s}$ ) at known coordinates at the external borders of the recorded regions. Then each animal was anesthetized and perfused as previously described (Rozzi et al., 2006). The brain was extracted, photographed, and cut (slice thickness, $60 \mu \mathrm{m}$ ). For both monkeys, each second and fifth section of a series of five was stained using the Nissl method (thionin, $0.1 \%$ in $0.1 \mathrm{M}$ acetate buffer, $\mathrm{pH} 3.7$ ). The locations of penetrations were then reconstructed on the basis of electrolytic lesions, stereotaxic coordinates, depths of penetrations, and functional properties. Subsequently, the cytoarchitectonic features of ventral premotor and inferior parietal areas were identified based on the criteria defined by Luppino and coworkers (Matelli et al., 1985; Gregoriou et al., 2006; Belmalih et al., 2009).

\section{Results}

We recorded 122 grasping neurons from the inferior parietal area PFG (Gregoriou et al., 2006) of both hemispheres of the two monkeys, and 89 grasping neurons from the posterior bank of the inferior arcuate sulcus and the adjacent convexity (area F5) (Matelli et al., 1985) of the right hemispheres of both monkeys (Fig. $2 A)$. All neurons were recorded during the performance of the complex action. In addition, all F5 neurons and 90\% of PFG neurons were recorded also during the simple action.

By comparing grasping neurons activity recorded during the complex action in the two experimental conditions (eating and placing), we found two basic neuronal categories (Tables 1, 2): no preference (NP) neurons, which did not show any difference in discharge intensity between the eating and placing conditions; and AGR neurons, which discharged differently during the eating and placing conditions. Among AGR neurons, we identified two different classes: neurons of the first class, defined as late preference (LP) neurons, showed their preference only during target grasping; the second class, defined as early preference (EP) neurons, discharged differently in the two conditions during both grasping of the lid and grasping of the target.

Figure $2 B$ shows the different proportion of these neuronal categories in the two areas. It is evident that AGR neurons were more frequently found in PFG than in F5 $\left(\chi^{2}=9.23, p<0.005\right)$, because of the presence of EP neurons, which were almost absent in area $\mathrm{F} 5\left(\chi^{2}=8.58, p<0.005\right)$.

\section{NP neurons encode motor acts regardless of the action goal}

Figure 3 shows examples of PFG and F5 NP neurons. Unit 204 (Fig. 3A) exemplifies the most frequent (55.1\%) behavior among NP neurons in PFG. The discharge of the neuron when the monkey grasps the target is similar in both simple and complex actions and, in the latter, a similar discharge is present during grasping of the lid regardless of the experimental condition (eating or placing). More than one-third (37.7\%) of F5 NP neurons showed this same behavior. Furthermore, in area F5, some NP neurons of this subclass $(N=13)$ were also active during grasping with the mouth (Fig. 3B). These grasping-with-hand-and-mouth neurons are virtually absent in area PFG.

Unit 76 (Fig. 3C) exemplifies the most frequent behavior of NP neurons in area F5. The neuronal discharge is not different between eating and placing conditions but, although it is always present during both grasping acts, it is significantly weaker during grasping of the lid than during grasping of the target in the complex action. Notably, neuronal activity during grasping of the target is similar during complex and simple action. The majority of F5 NP neurons (52.5\%) and a consistent proportion of those recorded in PFG (39.7\%) showed this behavior.

A correlation analysis performed on the whole NP neuronal population of both areas revealed that the discharges during grasping of the target in the simple and complex actions are sim-
Table 1. Neurons recorded in area PFG for each of the identified categories and their selectivity

\begin{tabular}{|c|c|c|c|c|c|c|}
\hline & \multicolumn{4}{|l|}{ AGR } & \multirow[b]{3}{*}{ NP } & \multirow[b]{3}{*}{ Total } \\
\hline & \multicolumn{2}{|l|}{ LP } & \multicolumn{2}{|l|}{ EP } & & \\
\hline & Eating & Placing & Eating & Placing & & \\
\hline Grasp $1>2$ & 4 & 2 & 3 & 1 & 3 & 13 \\
\hline Grasp $1=2$ & 14 & 1 & 4 & 5 & 32 & 56 \\
\hline Grasp $2>1$ & 24 & 2 & 4 & 0 & 23 & 53 \\
\hline Total & 42 & 5 & 11 & 6 & 58 & 122 \\
\hline
\end{tabular}

Grasp 1, Grasping the handle on the lid; Grasp 2, grasping the target.

Table 2. Neurons recorded in area F5 for each of the identified categories and their selectivity

\begin{tabular}{|c|c|c|c|c|c|c|}
\hline & \multicolumn{4}{|l|}{ AGR } & \multirow[b]{3}{*}{ NP } & \multirow[b]{3}{*}{ Total } \\
\hline & \multicolumn{2}{|l|}{$\mathrm{LP}$} & \multicolumn{2}{|l|}{ EP } & & \\
\hline & Eating & Placing & Eating & Placing & & \\
\hline Grasp $1>2$ & 4 & 1 & 1 & 0 & 6 & 12 \\
\hline Grasp $1=2$ & 0 & 3 & 0 & 0 & 23 & 26 \\
\hline Grasp $2>1$ & 11 & 7 & 0 & 1 & 32 & 51 \\
\hline Total & 15 & 11 & 1 & 1 & 61 & 89 \\
\hline
\end{tabular}

Grasp 1, Grasping the handle on the lid; Grasp 2, grasping the target.

ilar (F5: $t=1.68$, not significant; PFG: $t=0.46$, not significant) and positively correlated (F5: $r=0.93, p<0.001$; PFG: $r=$ $0.91, p<0.001)$.

Tables 1 and 2 clearly show that only a small fraction of NP neurons in both areas ( 3 of 58 in PFG, 6 of 61 in F5) discharged stronger during the first compared with the second grasping act. All these neurons were active, although weakly, during grasping of the target.

\section{LP neurons encode the action goal during the late stage of complex actions}

Figure 4, $A$ and $B$, shows examples of the different types of LP neurons recorded from area PFG and F5 during the complex action. Units 293 and 18 discharged stronger in the eating than in the placing condition during grasping of the target, whereas Units 346 and 41 exhibited the opposite selectivity, being more active during the placing condition. Notably, both these neurons discharged during grasping of the target but not during grasping of the handle of the lid. Six LP neurons in PFG and two in F5 showed this behavior. As a whole, more than half of LP neurons of both areas (55\% in PFG, 69\% in F5, $\chi^{2}=1.35$, not significant) were more strongly activated during grasping of the target compared with grasping of the lid. In the remaining LP neurons (Fig. $4 \mathrm{~A}$, Unit 354; $B$, Unit 9), the discharge during grasping of the lid was of similar intensity or even stronger.

Figure $4 C$ shows the time course and intensity of the discharge of the whole LP neuronal populations recorded from the two areas. A $2 \times 3$ repeated-measures ANOVA (factors, condition and epoch) followed by Newman-Keuls post hoc tests, evidenced that neuronal selectivity was present only during target grasping $(p<0.001$ for both areas), and this act was also associated with a stronger discharge compared with that observed during grasping of the lid ( $p<0.001$ for both areas). Furthermore, neuronal selectivity during target grasping, calculated in terms of the absolute value of PIs (see Materials and Methods, above), was higher in PFG $(\mathrm{PI}=0.36)$ than in F5 $(\mathrm{PI}=0.23)$ LP neuronal population $(t=2.45, p<0.05)$. 


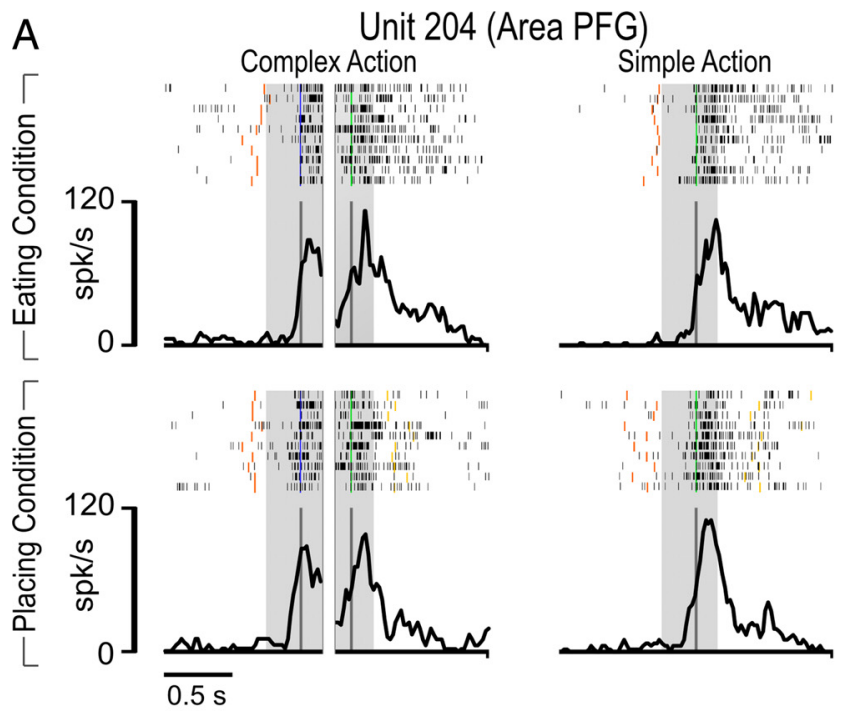

B

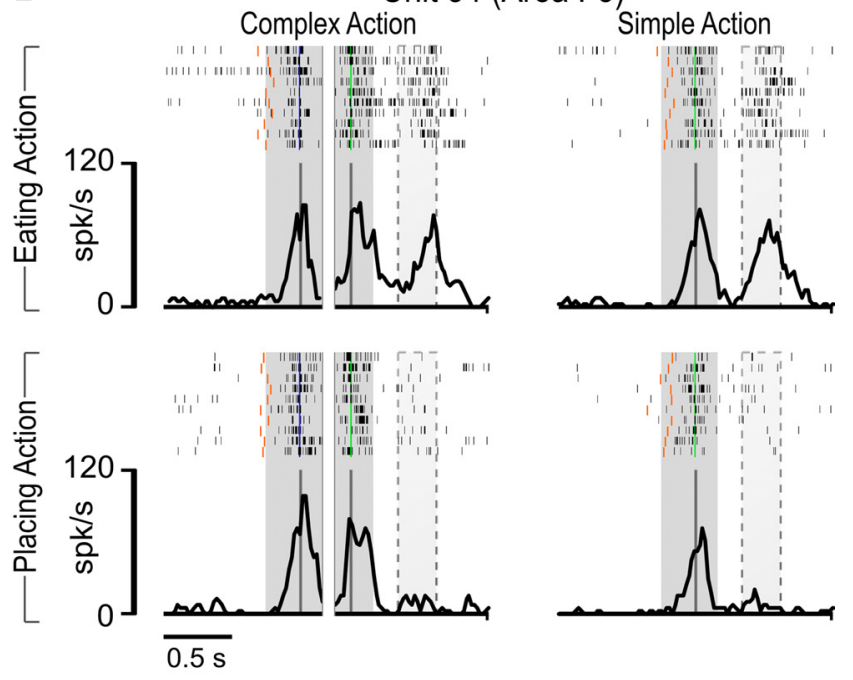

C

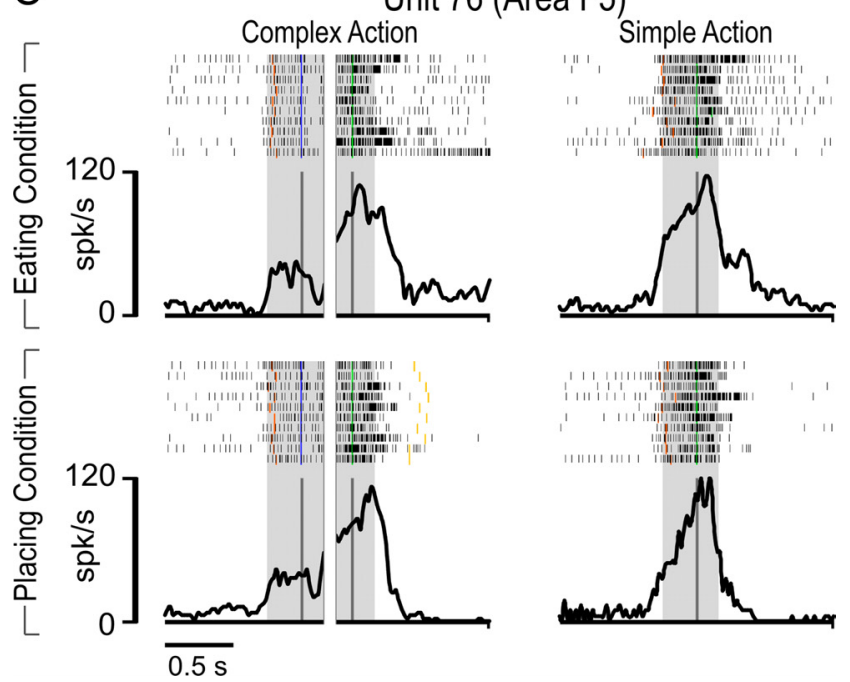

Figure 3. Example of NP neurons recorded in eating and placing conditions with simple and complex actions. $\boldsymbol{A}$, Example of a NP neuron of area PFG. The discharge does not differ between the eating and placing conditions, during either the grasping acts in complex action or target grasping in simple action. The gray shaded regions identify the two grasping epochs (first grasping of the lid, second grasping of the target). $\boldsymbol{B}$, Example of an NP hand-and-mouth grasping neuron of area F5. The dotted area approximates the period of time in which grasping
The higher discharge rate and neuronal preference during grasping of the target shown by LP neurons could depend on task complexity or the ordinal position of the grasping acts within the action sequence (Tanji, 2001). If this were the case, we could expect to observe differences in discharge rate or neuronal preference during grasping depending on whether the coded act was embedded into simple or complex actions. To explore this issue, we compared the firing rate and selectivity shown by single LP neurons in the complex action with that shown by the same neurons in the simple action. The example neuron shown in Figure $5 A$ was recorded from area PFG; it discharged strongly during target grasping in complex action, showing a clear preference for the eating compared with the placing condition. A similar firing rate and the same selectivity for the eating condition were evident when neuronal discharge was recorded during the simple action. An example of the response of an F5 LP neuron tested in the complex and simple actions is shown in Figure $5 B$. Figure $5 C$ shows that LP neurons firing rates during grasping of the target in complex and simple actions are positively correlated $(r=0.92$, $p<0.001$ ), even when considered separately for each area (PFG: $r=0.89, p<0.001$; F5: $r=0.94, p<0.001)$. Furthermore, neurons' firing rates are highly similar between the two tasks both in PFG (simple action, $46.0 \pm 25$ spikes/s; complex action, $47.2 \pm$ 26.5 spikes $/ \mathrm{s} ; t=0.65$, not significant) and F5 (simple action, $62.3 \pm 34 \mathrm{spikes} / \mathrm{s}$; complex action, $64.2 \pm 33.2 \mathrm{spikes} / \mathrm{s} ; t=0.81$, not significant), although it was higher for F5 than for PFG neurons during both simple $(t=2.27, p<0.05)$ and complex $(t=$ 2.33, $p<0.05)$ actions. As far as neuronal preference manifested in complex and simple actions is concerned, Figure $5 D$ shows that LP neurons' PIs calculated during grasping of the target in complex and simple actions are positively and significantly correlated $(r=0.86, p<0.001)$, even when considered separately for single areas (PFG: $r=0.83, p<0.001$; F5: $r=0.90, p<0.001$ ). Since simple and complex actions differ in terms of sequence of acts before target grasping but are identical afterwards, the similar preference and firing rate of LP neurons during target grasping in the two tasks rules out the possibility that their activity is influenced by task complexity or the ordinal position of the grasping acts within the action.

Another possibility is that the higher discharge rate and neuronal preference during target grasping in both simple and complex actions compared with the grasping of the lid in complex action depend on the fact that the reward was more immediate in the eating than in the placing condition, thus producing a different utility value (Glimcher, 2003; Schultz, 2004) for the two actions. To directly assess the possible impact of the reward on the discharge intensity of the grasping neurons included in the present study, we preliminarily tested with simple action neurons to be recorded with complex action, manipulating the action rewarding value. More specifically, we studied neurons' activity in the same condition (eating or placing) using different rewards to determine whether the reward used could affect the neuronal

$\leftarrow$

with the mouth occurred. Note that this response is present only in the eating condition. C, Example of an NP neuron of area F5. The discharge does not differ between the eating and placing condition, but it is higher during target grasping in both complex and simple actions compared with grasping of the lid in complex action. Shaded regions as in $\boldsymbol{A}$. Rasters and histograms are aligned with the moment when the monkey's hand touched the handle of the lid (blue bars) and when the monkey's hand touched the target inside the container (green bars). Other colored bars indicate the moment when monkey's hand detached from the starting position (red) and when it touched the border of the container in the placing condition (yellow). Note that in the placing condition, the target object was a small metallic solid (see Material and Methods). 


\section{A PFG neurons}

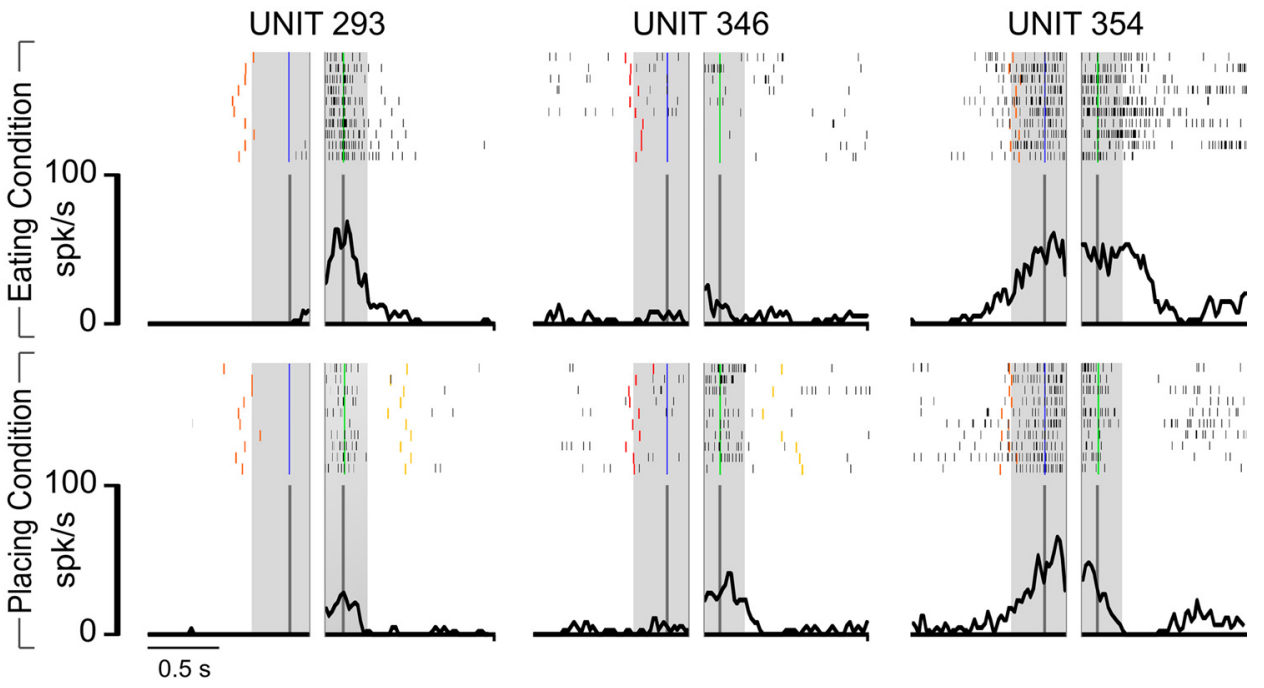

\section{B F5 neurons}
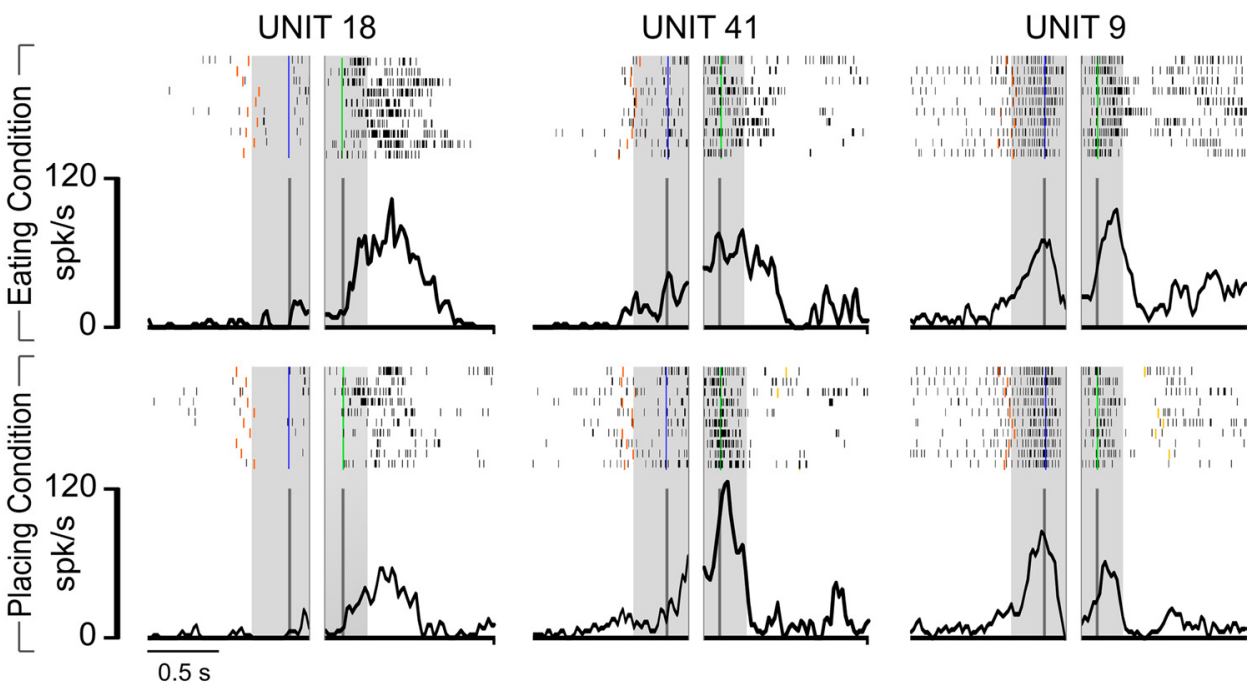

C

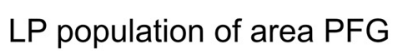

\section{LP population of area F5}


Figure 4. A, Activity of three LP neurons recorded in PFG with the complex action. B, Activity of three LP neurons recorded in F5 with the complex action. All conventions as in Figure 3 . Note that in the placing condition, the target object is a small metallic solid (see Material and Methods). C, Temporal profile of the net-normalized mean activity of the whole LP neuronal population of area PFG and area F5 in their preferred versus not-preferred condition. Neuronal activity is aligned on the first (left shaded region) and second (right shaded region) grasping act, as described for single neurons activity in Figure 3. Orange and gray shading indicate one SE.

preference. Figure 6 shows examples of neurons tested with different rewards. It is clear that varying the reward did not change neuronal preference for the eating (Fig. 6A) or placing (Fig. 6B) conditions, thus ruling out the possibility that a different rewarding or utility value of the two actions can account for the observed differential discharges. Together, these data suggest that the discharge of LP grasping neurons prospectively encodes the goal of the action in which grasping is embedded.
Although the goal of the action is known to the monkey even before starting action execution (Fig. 1, Task-cue), LP neurons appear to reflect this knowledge only during target grasping. In fact, no preference has been detected for any of the neurons included in this study during the baseline and premovement epochs. This suggests that sensory (visual/somatosensory) information related to the target to be grasped, which was available only after the container had been uncovered, is responsible for 


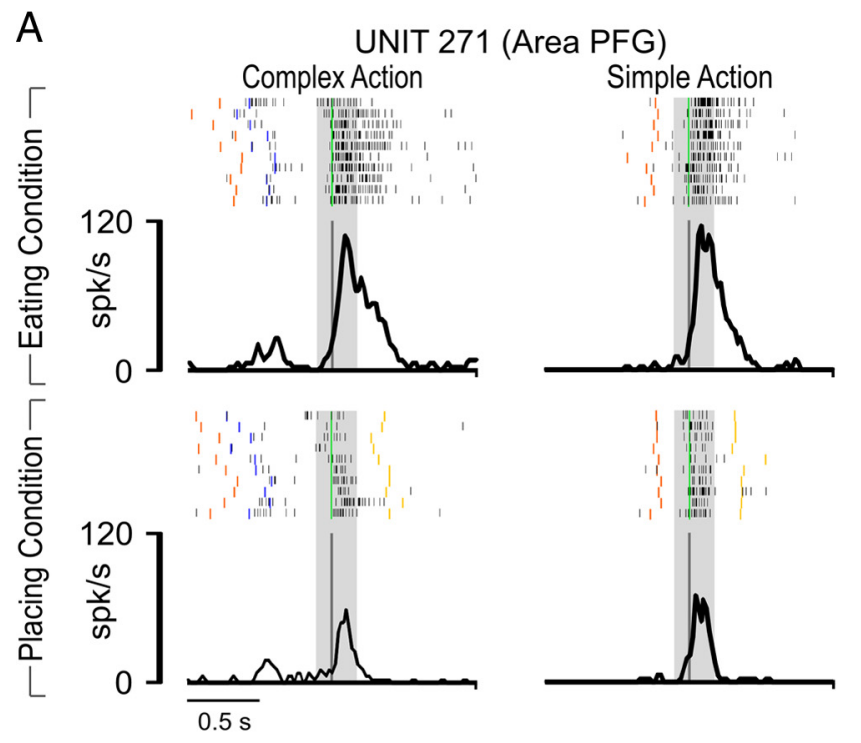

B

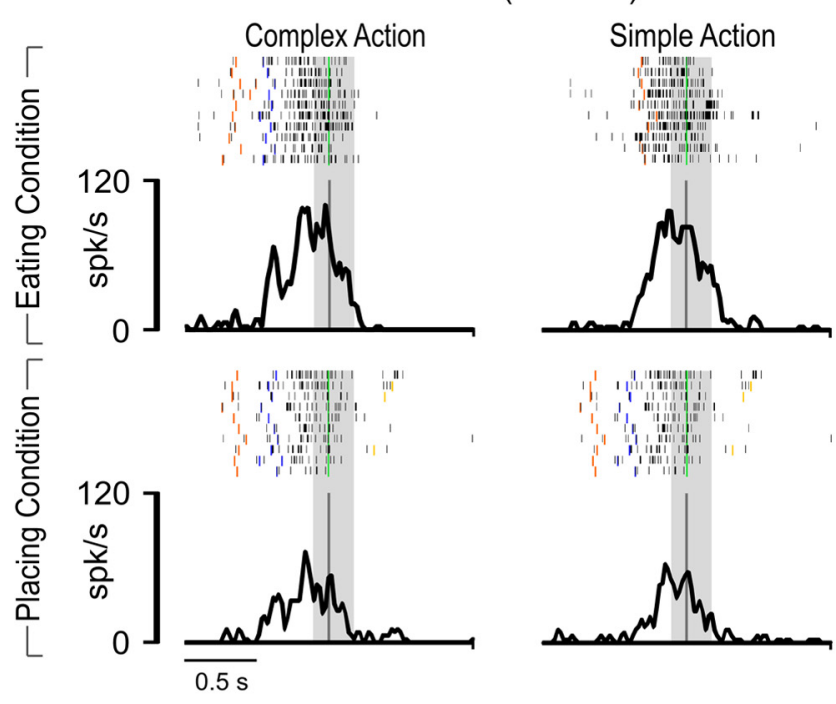

C
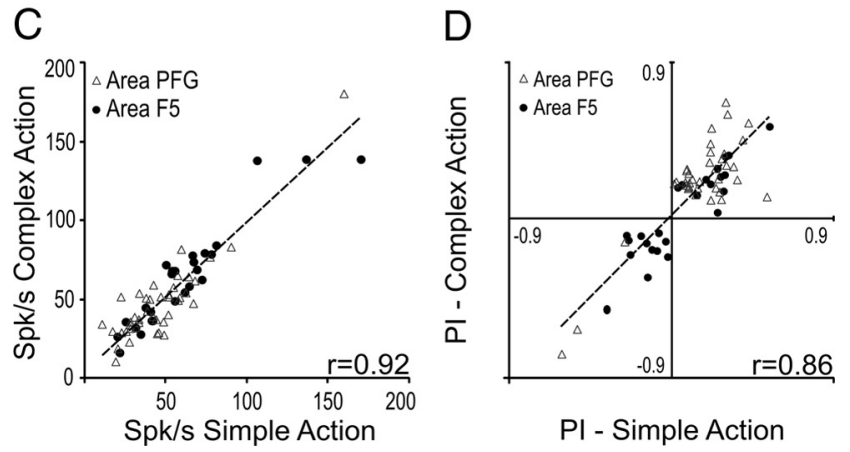

PI - Simple Action

Figure 5. A, Activity of a PFG LP neuron recorded in eating (top) and placing (bottom) conditions with both simple and complex actions. Note that for complex action, only the alignment on the second grasping is shown for comparative purposes. $\boldsymbol{B}$, Activity of a F5 LP neuron recorded in eating (top) and placing (bottom) conditions with both simple and complex action. Conventions for both $\boldsymbol{A}$ and $\boldsymbol{B}$ as in Figure 3 . Note that in the placing condition, the target object is a small metallic solid (see Material and Methods). $C$, Correlation between the average firing rate of LP neurons during grasping of the target (from $120 \mathrm{~ms}$ before to $160 \mathrm{~ms}$ after handtarget contact) in complex and simple action. $\boldsymbol{D}$, Correlation between LP neurons action goal preference expressed in terms of PI during grasping of the target in complex and simple action. the observed neuronal preference. This information could determine an enhancement of the discharge in the preferred condition or a decrease in the not-preferred condition. Interestingly, both these effects have been evidenced at the single neuron level (Fig. 4, Unit 354, Unit 9) and cannot be due to the type of target, per se. In fact, the same modulations (enhancement or decrease) have been observed in neurons tested in placing condition using the same food used in eating condition as the target (Fig. 7). Therefore, late preference appears to depend on the action goal to which the target has been associated.

\section{EP neurons reflect the final action goal since the early stage of the complex}

actions

Twenty-seven percent $(N=17)$ of PFG neurons influenced by the action goal showed their preference not only during target grasping, but also during grasping of the lid, despite contextual information cueing the action goal not being available. This type of neuron was almost absent in area F5 $(N=2)$; therefore we will focus only on EP neurons recorded in area PFG. In contrast to LP neurons, which most frequently discharge stronger during the second grasping act, most EP neurons (Table 1) discharged similarly during the two grasping acts or more strongly during the first one $\left(\chi^{2}=3.87, p<0.05\right)$

Figure $8 A$ shows examples of PFG EP neurons. Unit 212 discharged stronger in the eating than in the placing condition during both grasping the handle of the lid and grasping the food. Unit 237 showed the opposite behavior, being more strongly activated during both grasping acts in the placing condition than during the corresponding actions in the eating condition. Figure $8 B$ shows the time course and intensity of the activity of all EP neurons in their preferred condition (eating or placing). A $2 \times 3$ repeated-measures ANOVA (factors, condition and epoch) followed by Newman-Keuls post hoc tests evidenced that neuronal activity in the preferred condition was higher during both grasping acts compared with that during the not-preferred condition ( $p<0.001$ for both comparisons). Interestingly, none of the recorded neurons showed a preference only during grasping of the lid. Furthermore, neither EP single neurons nor the whole EP neuronal population displayed any preference during baseline or premovement epoch. A correlation analysis revealed that the preferences expressed by EP neurons on the two grasping acts are strongly and positively correlated $(r=0.904, p<0.001)$ (Fig. 8C).

\section{Early preference depends on memory-driven information about the final action goal}

To verify whether the early preference depends on an internally generated representation of the final goal based on previously acquired information about the target, we tested neurons with the complex action by using, at the beginning of each trial, an opaque screen instead of the transparent one (Fig. 1C), preventing the monkey from seeing which target (an object in the placing condition and a piece of food in the eating condition) had been placed in the container before the trial onset. Thus, the monkey could perform the action sequence, but ignored its final goal until the lid was removed and the target made visible. An example of a PFG grasping neuron recorded with the opaque screen is shown in Figure 9A. When tested with the transparent screen, this neuron discharged stronger in the eating compared with the placing condition during both grasping acts (EP neuron) (Fig. 9A, left). When the opaque screen was used (Fig. $9 A$, middle), the neuron lost its early preference during grasping of the handle of the lid 
A

UNIT 238

Grasp apple to eat

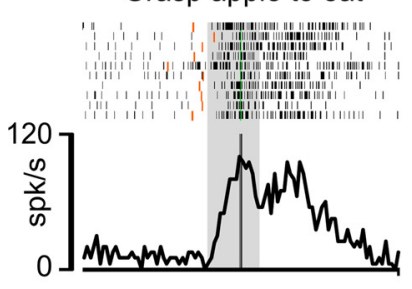

Grasp candy to eat

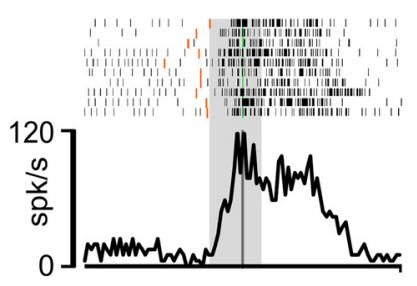

Grasp apple to place - reward: candy -

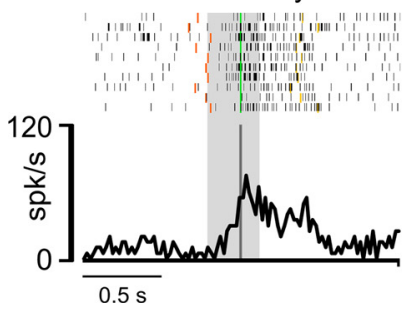

B

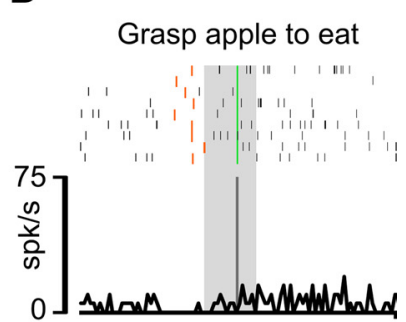

Grasp cube to place - no reward -

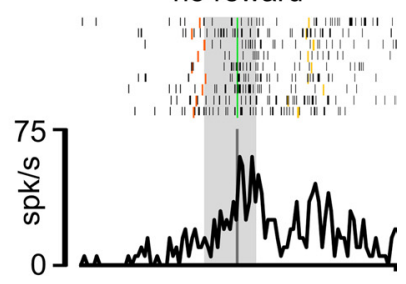

Grasp apple to place - reward: candy -

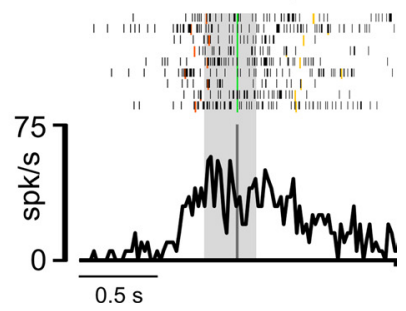

Figure 6. Examples of PFG grasping neurons recorded with simple grasp-to-eat and grasp-to-place actions using different rewards and target objects. $A$, Activity of a neuron selective for the eating condition in simple action. When grasping a piece of apple or a candy (the latter is systematically preferred by the monkey), the neuron firing rate remains constant and higher than that observed during the grasping of a piece of apple to place it, despite using candy as a reward for correct task accomplishment. $\boldsymbol{B}$, Activity of a neuron selective for placing condition in simple action. Note that, even in this case, the neuronal selectivity for the placing condition remained the same both when grasping a piece of apple to place, obtaining candy as a reward, and when grasping a metallic cube for placing, despite that no reward was delivered in this condition. Conventions as in Figure 3.

\section{LP population of area PFG}

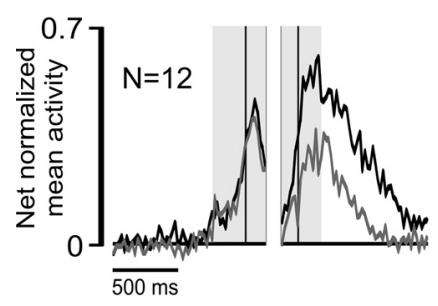

LP population of area F5

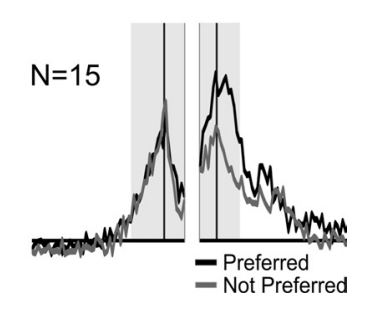

Figure 7. Temporal profile of the net-normalized activity of LP neuronal subpopulations of area PFG (left) and F5 (right) in their preferred and not-preferred conditions, tested by using an identical piece of food as a target in both the eating and placing conditions. Neuronal activity is aligned on the first (left) and second (right) grasping act, as in Figure 4C.

but, once the lid was removed, the target (either food or object) became visible and the neuron preference for eating was restored during grasping of the target. This neuron was further tested with the opaque screen, but in this case the trials were run in blocks (Fig. 9A, right). Here, in each trial the monkey could predict the final action goal solely based on its memory of the previously executed trials. It is clear that neuronal preference is maintained despite the lack of direct visual information cueing the action

goal. All EP neurons tested with opaque screen in blocks $(N=5)$ confirmed this effect.

Ten neurons (five selective for eating and five for placing conditions, recorded from both monkeys) were recorded long enough to be tested during task execution with both the transparent and opaque screen (randomizing the eating and placing trials). A $2 \times 2 \times 3$ repeated-measures ANOVA (factors, screen, condition, and epoch), followed by Newman-Keuls post hoc tests, confirmed the effect described at the single neuron level (Fig. 9B). Interestingly, a significant interaction between the screen and condition factors $\left(F_{(1,9)}=15.9, p<0.005\right)$ revealed that when contextual information was lacking (Fig. $9 B$, opaque screen), the activity associated with the preferred condition was reduced $(p<0.05)$, whereas that associated with the not-preferred one was increased $(p<0.05)$ compared with transparent screen condition.

To verify that when the opaque screen was used the monkey could not have access to any cue for selecting, at the beginning of the task, a specific action sequence, we compared movement onset latency in the eating and placing conditions when transparent and opaque screens were used (Fig. 9C). A $2 \times 2$ repeatedmeasures ANOVA (factors, screen and condition) revealed that the use of the opaque screen determined an increase of movement-onset latency in both conditions $\left(F_{(1,53)}=45.6, p<\right.$ $0.001)$ and an interaction between screen and condition $\left(F_{(1,53)}=\right.$ $8.78, p<0.005)$. More specifically, Newman-Keuls post hoc tests evidenced that the reaction time was shorter in the eating than in the placing condition $(p<0.001)$, but this difference disappeared when the opaque screen was applied. Differently from the time required for action selection, as revealed by the movement onset latency, the time required for executing the first part of the two action sequences should not be different across the conditions. To verify this, we performed a further $2 \times 2$ repeated-measures ANOVA (factors, screen and condition) on reaching time and grasping-the-lid time. This analysis showed only a significant main effect of the factor screen (reaching time, $F_{(1,53)}=28.6, p<0.001$; graspint-the-lid tme, $\left.F_{(1,53)}=7.52, p<0.01\right)$, evidencing that the monkey was slower in performing the task when the opaque screen was used, but behaved similarly in the two conditions.

\section{Discussion}

In this study, we demonstrate that action goal-related neurons, as previously characterized using simple actions (Fogassi et al., 2005; Bonini et al., 2010), can be further distinguished into two different categories: LP and EP neurons. LP neurons reflect a specific action goal only in the late phase of action unfolding, whereas EP neurons encode the action goal already during the early phase of the action sequence. We will first briefly discuss the properties of neurons not influenced by the action goal (NP neurons). Then, we will focus on the relative contribution of sensory information and the agent's motor intention to the functional properties of LP and EP neurons.

\section{No preference neurons: abstract coding of single motor acts} and motor synergies for actions

$\mathrm{NP}$ neurons are more frequently found in area F5 than in PFG, and can be subdivided into two categories.

First, there are neurons discharging similarly during every grasping act, both in simple and complex actions. Some of them, only in area F5, also responded during mouth grasping (Rizzolatti et al., 1988), thus coding grasping in an abstract fashion. This could reduce the computational load for motor control (Prabhu et al., 2009) and may be particularly important when an individual has to perform a motor act included in his/her motor reper- 




\section{B EP neuronal population}
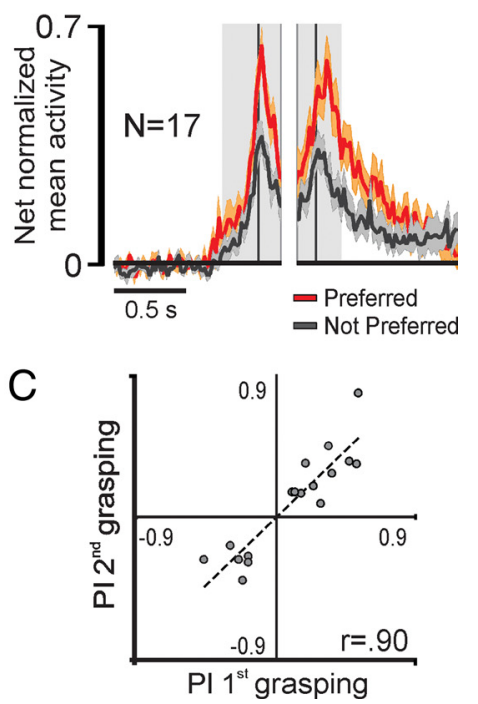

Figure 8. $A$, Activity of a PFG EP neuron recorded with complex action. Conventions as in Figure 3. Note that in the placing condition, the target object was a small metallic solid (see Material and Methods). B, Temporal profile of the net-normalized activity of the whole EP neuronal population of area PFG in their preferred versus not-preferred condition. Conventions as in Figure 4B. C, Correlation between EP neurons' PIs, calculated for the first and the second grasping acts of the sequence. Note that positive and negative PI values indicate a preference for eating and placing condition, respectively.

toire, but using a different movement pattern, for example, during grasping with a tool (Umiltà et al., 2008).

Second, other NP neurons responded more strongly during target grasping in both complex and simple actions compared with grasping of the lid. The preference for target grasp occurred despite the fact that hand shaping and the actual grasping phases preceding lid removal were the same (Fig. $3 C$ ). The difference in discharge rate between lid and target grasping could be explained by a possible link between these neurons and those encoding subsequent motor acts (i.e., arm flexion for bringing) following target, but not lid, grasping. This idea is in agreement with the results of long-train microstimulation studies (Graziano et al., 2002) and parallels the general organization of somatosensory receptive fields of ventral premotor cortex (Rizzolatti et al., 1981a,b) and inferior parietal lobule (Yokochi et al., 2003; Rozzi et al., 2008). In these areas, sensory-motor associations at the single neuron level could facilitate the link between successive motor acts such as grasping/bringing or bringing/biting. This class of NP neurons could thus be important to organize specific motor synergies that, in turn, can constitute multipurpose building blocks for structuring simple actions, regardless of their final goal.

A few NP neurons are more strongly activated during grasping of the lid. This could suggest that such neurons encode grasp-toremove, but the presence of a clear response during the successive grasping of the target and the features of the task, not aimed at controlling this variable, prevent us from reaching a final conclusion.

\section{Late preference neurons: sensory-cued prospective encoding of action goal}

LP neurons have been found both in PFG and F5, and are characterized by a differential discharge between the experimental conditions during target, but not lid, grasping.

Motor parameters such as grip type (Raos et al., 2006) or sensory feedback from the grasped object could not be responsible for the observed preference, since the monkey always used the same type of grip and, in most cases, grasped the same target object in both experimental conditions. Furthermore, we showed that the manipulation of reward or action utility value (Glimcher, 2003; Schultz, 2004) did not affect neurons selectivity. Notably, the same firing pattern and selectivity could be obtained by recording LP neurons during the simple action. Thus, the differential discharges of LP neurons reflect the final action goal, although exclusively during the grasping act that immediately leads to its accomplishment.

Action goal preference could derive from facilitatory or inhibitory effects. In fact, taking as a reference the discharge rate of LP neurons during grasping of the lid, some of them increase (Fig. 4, Unit 293, Unit 346) whereas others decrease (Fig. 4, Unit 354) their activity during target grasping in one of the two conditions (the preferred and not-preferred, respectively). Since these modulations occur only when the monkey can see the target and interact with it, the late action goal preference shown by these neurons very likely depends on the availability of sensory information about the target (food or object) that has been associated to a specific goal.

LP neurons could contribute to organizing actions by facilitating other neurons coding successive motor acts not only in terms of the required motor synergies, but based on the action final goal. Indeed, previous studies demonstrated that grasp-toplace neurons maintain the same action goal selectivity regardless of whether placing is performed with forearm flexion or abduction (Fogassi et al., 2005).

Notably, PFG LP neurons display a stronger action goal preference than those of F5, confirming previous findings (Bonini et al., 2010). The parietal cortex thus appears to play a leading role in organizing the final stages of complex actions.

\section{Early preference neurons reflect the motor intention of the acting individual}

EP neurons have been found almost exclusively in area PFG. They discharge differently during grasping of the lid and the target in complex action, and the magnitudes of their preferences during both grasping acts are similar and positively correlated. These data suggest that the same sources of afferent information determine this effect. What is this information? 

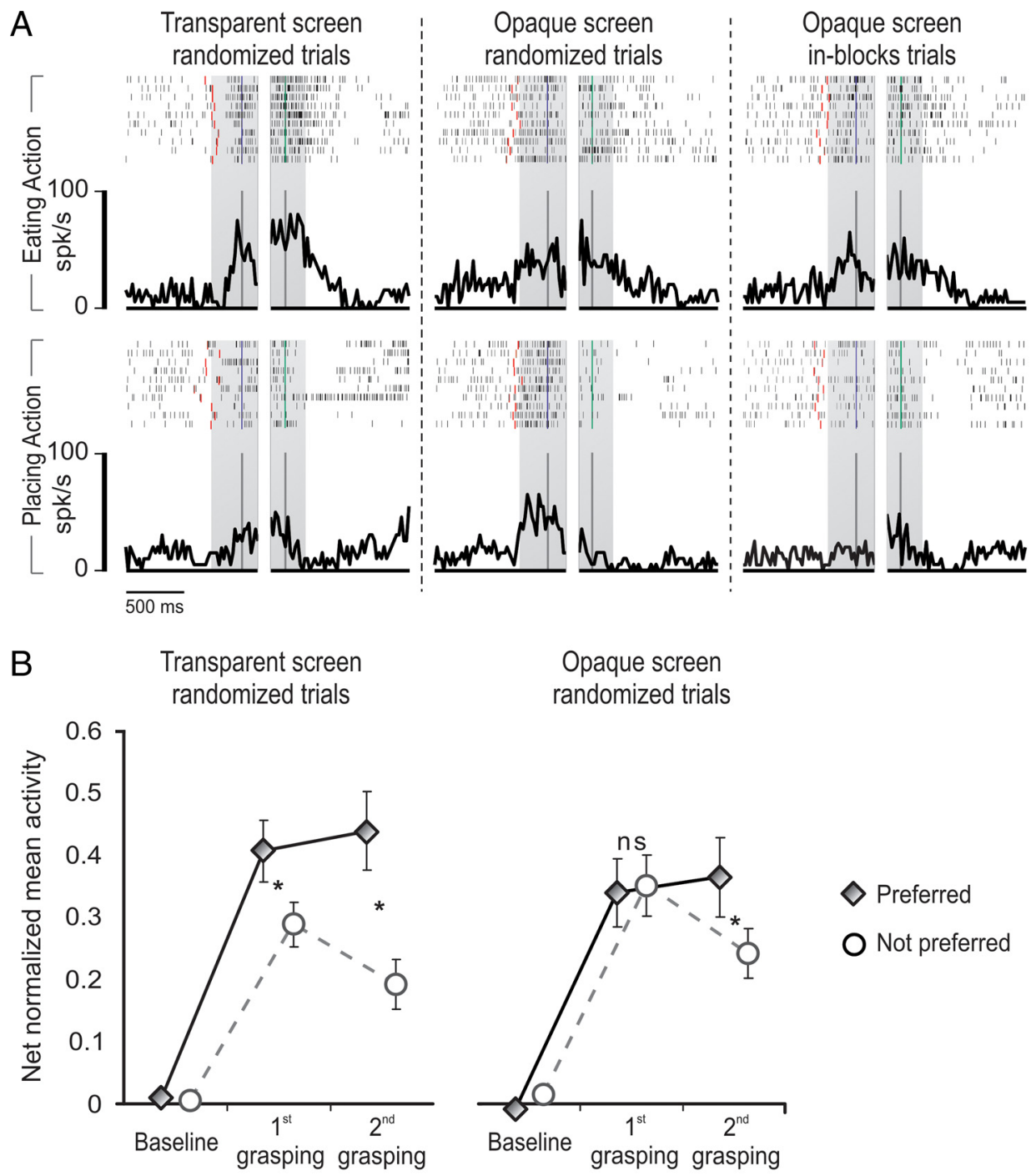

C

Movement onset latency

Reaching time

Actual grasping time
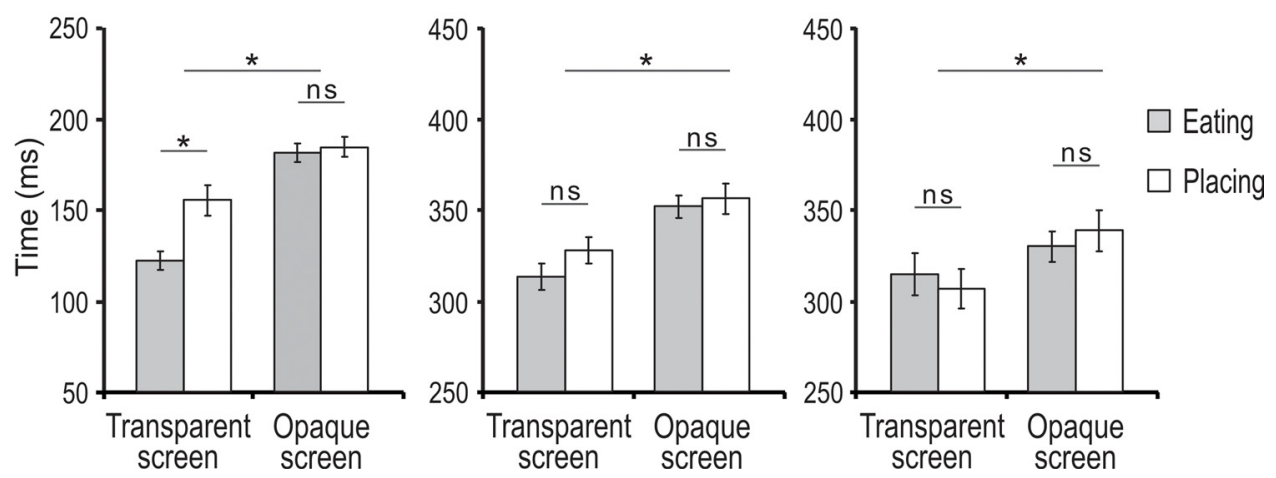

Figure 9. $\quad \boldsymbol{A}$, Example of an EP neuron tested in the motor task with both transparent and opaque screens. Right, Neuron discharge with an opaque screen with trials run in-blocks, enabling the monkey to predict the final action goal based on the memory of previously executed trials. Note that, in this condition, the early selectivity is still present. Conventions as in Figure 3 . $\boldsymbol{B}$, Comparison between the net-normalized mean activity during the first and the second grasping epochs, recorded from 10 neurons tested in the complex action with both transparent and opaque screens. $\boldsymbol{C}$, Comparison between movement onset latency (left), reaching time (center), and grasping time (right) of the two monkeys during the execution of eating and placing conditions of the complex action run with transparent and opaque screens. Error bars indicate the SE. ns, Not significant. ${ }^{*} p<0.05$.

Since grasping of the lid always occurs in the same environmental context, we can exclude the hypothesis that early action goal preference derives from sensory information, as proposed for LP neurons. Instead, our findings strongly suggest that the modulation of EP neurons is driven by an internally generated representation of the action goal specifying why the action is performed, which is the motor intention of the acting individual (Rizzolatti et al., 2006). Thus, our data support the view that the 
intention does not terminate with movement onset, but is kept in mind and guides action unfolding until its completion (Pacherie, 2008). Early preference appears, indeed, only during actual performance of motor acts (grasping) and not during movement selection or motor preparation, as previously reported for neurons in other regions of the posterior parietal cortex (Godschalk and Lemon, 1989; Cui and Andersen, 2007).

In our study, the monkey's intention of eating or placing can be established only when contextual information is available before action onset (i.e., transparent screen trials), or when action goal can be inferred based on the memory of previously executed trials (i.e., opaque screen trials run in blocks). When this information is lacking (i.e., randomized opaque screen trials), EP neurons lose their early intentional selectivity. However, the monkey can still perform the first part of the action relying on the learned motor sequence (Tanji, 2001) and the remaining sensory information (Murata et al., 2000; Buneo et al., 2002; Rozzi et al., 2008; Fattori et al., 2010). Interestingly, neurons do not cease firing, showing a discharge rate that is intermediate between that shown in their preferred and not-preferred conditions run with the transparent screen. This suggests that, under uncertainty, EP neurons are recruited regardless of their action-goal selectivity, in accordance with the findings previously reported for directional selectivity of dorsal premotor-reaching neurons (Cisek and Kalaska, 2005) or grip selectivity of parietal neurons (Baumann et al., 2009). After the monkey removes the lid, the sight of the target enables it to establish the goal, which is reflected in the neurons recovery of their intentional specificity during the successive grasping act.

\section{Conclusions}

The present study suggests that ventral premotor and inferior parietal neurons can code the goal of intentional actions at different levels of motor abstraction. On one side, LP neurons display a prospective encoding of the action goal, very likely facilitating subsequent acts. On the other side, EP neurons reflect the action goal at higher level of abstraction, keeping active the representation of the individual's motor intention during action unfolding.

A number of studies have assigned a major role to the prefrontal cortex in sequential aspects of motor cognition, such as the acquisition of motor sequences (Koechlin et al., 2002; Averbeck et al., 2006) and the pursuit of behavioral goals (Charron and Koechlin, 2010). Furthermore, prefrontal neurons have been described encoding action categories (Shima et al., 2007; Tanji et al., 2007) and behavioral goals (Saito et al., 2005) at different level of abstraction, but not the actual movements of a specific effector (Mushiake et al., 2006). However, recent fMRI studies on humans suggest that the inferior parietal cortex is also involved in high order sequential motor cognition (Jubault et al., 2007), very likely based on its rich anatomical connections with prefrontal regions (Divac et al., 1977; Petrides and Pandya, 1999; Rozzi et al., 2006).

Altogether, the present and previous data favor the idea that parieto-premotor circuits work together with the prefrontal cortex to organize motor acts into action sequences and to keep active internal representations of the individual's motor intention.

\section{References}

Averbeck BB, Sohn JW, Lee D (2006) Activity in prefrontal cortex during dynamic selection of action sequences. Nat Neurosci 9:276-282.

Baumann MA, Fluet MC, Scherberger H (2009) Context-specific grasp movement representation in the macaque anterior intraparietal area. J Neurosci 29:6436-6448.

Belmalih A, Borra E, Contini M, Gerbella M, Rozzi S, Luppino G (2009)
Multimodal architectonic subdivision of the rostral part (area F5) of the macaque ventral premotor cortex. J Comp Neurol 512:183-217.

Bonini L, Rozzi S, Serventi FU, Simone L, Ferrari PF, Fogassi L (2010) Ventral premotor and inferior parietal cortices make distinct contribution to action organization and intention understanding. Cereb Cortex 20:1372-1385.

Botvinick MM (2007) Multilevel structure in behaviour and in the brain: a model of Fuster's hierarchy. Philos Trans R Soc Lond B Biol Sci 362:1615-1626.

Buneo CA, Jarvis MR, Batista AP, Andersen RA (2002) Direct visuomotor transformations for reaching. Nature 416:632-636.

Charron S, Koechlin E (2010) Divided representation of concurrent goals in the human frontal lobes. Science 328:360-363.

Chen J, Reitzen SD, Kohlenstein JB, Gardner EP (2009) Neural representation of hand kinematics during prehension in posterior parietal cortex of the macaque monkey. J Neurophysiol 102:3310-3328.

Cisek P, Kalaska JF (2005) Neural correlates of reaching decisions in dorsal premotor cortex: specification of multiple direction choices and final selection of action. Neuron 45:801-814.

Cui H, Andersen RA (2007) Posterior parietal cortex encodes autonomously selected motor plans. Neuron 56:552-559.

Divac I, Lavail JH, Rakic P, Winston KR (1977) Heterogeneous afferents to the inferior parietal lobule of the rhesus monkey revealed by the retrograde transport method. Brain Res 123:197-207.

Fattori P, Raos V, Breveglieri R, Bosco A, Marzocchi N, Galletti C (2010) The dorsomedial pathway is not just for reaching: grasping neurons in the medial parieto-occipital cortex of the macaque monkey. J Neurosci 30:342-349.

Fogassi L, Ferrari PF, Gesierich B, Rozzi S, Chersi F, Rizzolatti G (2005) Parietal lobe: from action organization to intention understanding. Science 308:662-667.

Fuster JM (2006) The cognit: a network model of cortical representation. Int J Psychophysiol 60:125-132.

Fuster JM (2008) The prefrontal cortex, 4 th edition. London: Academic.

Gardner EP, Babu KS, Reitzen SD, Ghosh S, Brown AS, Chen J, Hall AL, Herzlinger MD, Kohlenstein JB, Ro JY (2007) Neurophysiology of prehension. I. Posterior parietal cortex and object-oriented hand behaviors. J Neurophysiol 97:387-406.

Glimcher PW (2003) The neurobiology of visual-saccadic decision making. Annu Rev Neurosci 26:133-179.

Godschalk M, Lemon RN (1989) Preparation of visually cued arm movements in monkey: involvement of inferior parietal cortex. Brain Behav Evol 33:122-126.

Graziano MS, Taylor CS, Moore T (2002) Complex movements evoked by microstimulation of precentral cortex. Neuron 34:841-851.

Gregoriou GG, Borra E, Matelli M, Luppino G (2006) Architectonic organization of the inferior parietal convexity of the macaque monkey. J Comp Neurol 496:422-451.

Jeannerod M (1988) The neural and behavioural organization of goaldirected movements. Oxford: Oxford UP.

Jubault T, Ody C, Koechlin E (2007) Serial organization of human behavior in the inferior parietal cortex. J Neurosci 41:11028-11036.

Kakei S, Hoffman DS, Strick PL (2003) Sensorimotor transformations in cortical motor areas. Neurosci Res 46:1-10.

Koechlin E, Jubault T (2006) Broca's area and the hierarchical organization of human behavior. Neuron 50:963-974.

Koechlin E, Danek A, Burnod Y, Grafman J (2002) Medial prefrontal and subcortical mechanisms underlying the acquisition of motor and cognitive action sequences in humans. Neuron 35:371-381.

Mason CR, Theverapperuma LS, Hendrix CM, Ebner TJ (2004) Monkey hand postural synergies during reach-to-grasp in the absence of vision of the hand and object. J Neurophysiol 91:2826-2837.

Matelli M, Luppino G, Rizzolatti G (1985) Patterns of cytochrome oxidase activity in the frontal agranular cortex of the macaque monkey. Behav Brain Res 18:125-136.

Murata A, Gallese V, Luppino G, Kaseda M, Sakata H (2000) Selectivity for the shape, size, and orientation of objects for grasping in neurons of monkey parietal area AIP. J Neurophysiol 83:2580-2601.

Mushiake H, Saito N, Sakamoto K, Itoyama Y, Tanji J (2006) Activity in the lateral prefrontal cortex reflects multiple steps of future events in action plans. Neuron 50:631-641.

Pacherie E (2008) The phenomenology of action: a conceptual framework. Cognition 107:179-217. 
Petrides M, Pandya DN (1999) Dorsolateral prefrontal cortex: comparative cytoarchitectonic analysis in the human and the macaque brain and corticocortical connection patterns. Eur J Neurosci 11:1011-1036.

Prabhu G, Shimazu H, Cerri G, Brochier T, Spinks RL, Maier MA, Lemon RN (2009) Modulation of primary motor cortex outputs from ventral premotor cortex during visually guided grasp in the macaque monkey. J Physiol 587:1057-1069.

Raos V, Umiltá MA, Murata A, Fogassi L, Gallese V (2006) Functional properties of grasping-related neurons in the ventral premotor area F5 of the macaque monkey. J Neurophysiol 95:709-729.

Rizzolatti G, Scandolara C, Matelli M, Gentilucci M (1981a) Afferent properties of periarcuate neurons in macaque monkeys. I. Somatosensory responses. Behav Brain Res 2:125-146.

Rizzolatti G, Scandolara C, Matelli M, Gentilucci M (1981b) Afferent properties of periarcuate neurons in macaque monkeys. II. Visual responses. Behav Brain Res 2:147-163.

Rizzolatti G, Camarda R, Fogassi L, Gentilucci M, Luppino G, Matelli M (1988) Functional organization of inferior area 6 in the macaque monkey. II. Area F5 and the control of distal movements. Exp Brain Res 71:491-507.

Rizzolatti G, Ferrari PF, Rozzi S, Fogassi L (2006) The inferior parietal lobule: where action becomes perception. Novartis Found Symp 270:129-140.

Rozzi S, Calzavara R, Belmalih A, Borra E, Gregoriou GG, Matelli M, Luppino G (2006) Cortical connections of the inferior parietal cortical convexity of the macaque monkey. Cereb Cortex 16:1389-1417.

Rozzi S, Ferrari PF, Bonini L, Rizzolatti G, Fogassi L (2008) Functional organization of inferior parietal lobule convexity in the macaque monkey: electrophysiological characterization of motor, sensory and mirror responses and their correlation with cytoarchitectonic areas. Eur J Neurosci 28:1569-1588.

Saito N, Mushiake H, Sakamoto K, Itoyama Y, Tanji J (2005) Representation of immediate and final behavioral goals in the monkey prefrontal cortex during an instructed delay period. Cereb Cortex 15:1535-1546.

Salinas E (2004) Context-dependent selection of visuomotor maps. BMC Neurosci 5:47.

Schultz W (2004) Neural coding of basic reward terms of animal learning theory, game theory, microeconomics and behavioural ecology. Curr Opin Neurobiol 14:139-147.

Shima K, Isoda M, Mushiake H, Tanji J (2007) Categorization of behavioural sequences in the prefrontal cortex. Nature 445:315-318.

Tanji J (2001) Sequential organization of multiple movements: involvement of cortical motor areas. Annu Rev Neurosci 24:631-651.

Tanji J, Shima K, Mushiake H (2007) Concept-based behavioral planning and the lateral prefrontal cortex. Trends Cogn Sci 11:528-534.

Toth LJ, Assad JA (2002) Dynamic coding of behaviourally relevant stimuli in parietal cortex. Nature 415:165-168.

Umiltà MA, Escola L, Intskirveli I, Grammont F, Rochat M, Caruana F, Jezzini A, Gallese V, Rizzolatti G (2008) When pliers become fingers in the monkey motor system. Proc Natl Acad Sci U S A 105:2209-2213.

Yokochi H, Tanaka M, Kumashiro M, Iriki A (2003) Inferior parietal somatosensory neurons coding face-hand coordination in Japanese macaques. Somatosens Mot Res 20:115-125. 